\section{$\$$ Research Square \\ Preprints are preliminary reports that have not undergone peer review. \\ They should not be considered conclusive, used to inform clinical practice, or referenced by the media as validated information.}

\title{
The efficacy and safety of immune checkpoints inhibitors plus radiotherapy as a combination therapy for advanced non-small cell lung carcinoma: a meta- analysis
}

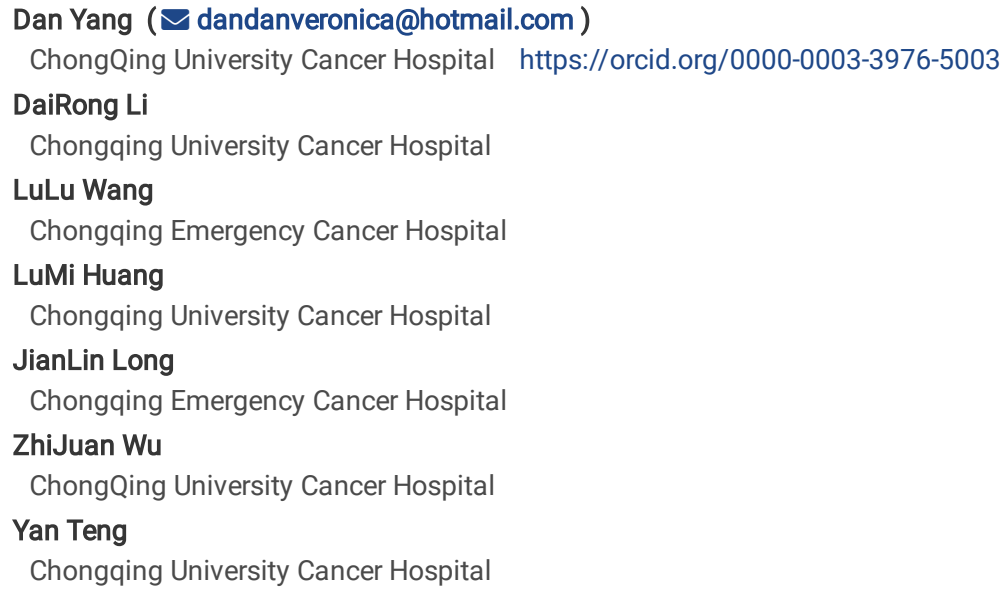

Keywords: Immune checkpoint inhibitor, Programmed death 1, Programmed death 1 ligand 1, cytotoxic T-lymphocyte-associated antigen 4, Radiotherapy, Nonsmall cell lung cancer

Posted Date: August 20th, 2020

DOI: https://doi.org/10.21203/rs.3.rs-62263/v1

License: (c) (7) This work is licensed under a Creative Commons Attribution 4.0 International License. Read Full License 


\section{Abstract}

\section{Background}

Immune checkpoint inhibitors have showed good efficacy in advanced non-small-cell lung cancer(NSCLC). This meta-analysis was conducted to explore the therapeutic efficacy and safety of immune checkpoint inhibitors combined with radiotherapy for patients with advanced NSCLC.

\section{Methods}

We used many proper keywords to perform a systematic search in databases and performed a meta-analysis of trials that conducted immune checkpoint inhibitors plus radiotherapy as a treatment for advanced non-small-cell lung cancer. The outcomes included objective response rate (ORR), disease control rate (DCR), progression-free survival (PFS), overall survival (OS) and treatment-related adverse events. A fixed-effect or random-effects model was adopted depending on between-study heterogeneity.

\section{Results}

Ten trials were finally included in this meta-analysis. The combined ORR and DCR was 34\% (19\%-49\%) and 72\% (65\%-79\%). The combined six-month progression-free survival rates (PFSR6m) and one-year overall survival rate(OSR1y) were $50.0 \%(29.4 \%-72.8 \%)$ and $59.0 \%$ (48\%-69\%). Immune checkpoint inhibitors plus radiotherapy was significantly associated with improvement of PFS (hazards ratio [HR], $0.64 ; 95 \% \mathrm{Cl} 0.46-0.90 ; \mathrm{P}=0.01$ ), OS (HR, $0.62 ; 95 \% \mathrm{Cl}$ $0.46-0.85 ; \mathrm{P}=0.003)$. In subgroup analyses, the combined ORR was $31.5 \%(16.4 \%-46.6 \%)$, combined DCR was $72.1 \%(63.3 \%-80.9 \%)$, combined PFS6m was $50.0 \%(20.6 \%-79.5 \%)$ and combined OSR1y was 59.7\% (48.1\%-71.2\%) for anti-PD-1/PD-L1 antibody plus radiotherapy. The combined ORR was $19.5 \%$ (9.1\%-29.8\%) for anti-CTLA4 antibody plus radiotherapy. The combined PFS6m and OSR1y were 58.9\% (26.2\%-91.7\%) and 59.7\% (29.7\%-89.8\%) respectively for the concurrent therapy group. And the combined PFS6m and OS1y were 55.2\% (41.6\%-68.8\%) and 55.7\% (42.1\%-69.3\%) for sequential therapy. For the lowdose radiotherapy group. The combined PFS6m and OS1y were $45.9 \%$ (27.0\%-64.8\%) and 51.6\% (37.5\%-65.6\%). The combined PFS6m and 0S1y were 71.1\% (37.9\%-104.4\%) and $67.4 \%$ (29.2\%-105.6\%) separately for high-dose radiotherapy group. When using as first-line therapy. The combined ORR was 53.8\% (23.6\%-84.1\%) and combined ORR was 76.5\% (65.0\%-88.1\%). While as subsequent therapy, the combined ORR and DCR were $25.9 \%$ (14.7\%-37.2\%) and $69.9 \%$ (60.9\%-78.9\%). The combined PFS6m and OS1y were 51.1\% (40.3\%-61.9\%) and 51.8\% (40.9\%-62.7\%).

\section{Conclusions}

Immune checkpoint inhibitors plus radiotherapy might be an effective and tolerable option as a treatment for advanced NSCLC.

\section{Background}

Lung cancer is still a worldwide healthy problem, leading the highest mortality for both male and female ${ }^{[1]}$. In China, the data from cancer statistics showed that there were 733,000 newly diagnosed lung cancer cases and 60 million lung cancer deaths in 2015 ${ }^{[2]}$. NSCLC took the most proportion in all types of lung cancer, about $85-90 \%{ }^{[3]}$. Unfortunately, most patients were at advanced stage when diagnosed, no chance of healing ${ }^{[4]}$. These patients had to rely on systemic therapy. In which, the traditional platinum-based chemotherapy was the most important treatment in a very long period. But the survival time was unsatisfactory for its efficacy was limited and its toxic side's effect was remarkably ${ }^{[5]}$. A significant improvement has been achieved by gene-driven targeted therapy, including epidermal growth factor receptor (EGFR)-Tyrosine Kinase Inhibitor (TKI), inhibitor of anaplastic lymphoma kinase (ALK) and ROS protooncogene 1 receptor tyrosine kinase (ROS1). The gene-driven targeted therapy was reported a much higher response rate for patients with relative gene-driven mutation ${ }^{[6-8]}$. Moreover, it reveals Chinese patients gained more benefits because of a higher mutation rate in EGFR ${ }^{[9]}$. However, for patients without relative gene-driven mutation, there was still no better choice of treatment.

Until the past decades, the appearance of immune checkpoint inhibitors (ICls) was an inspiring breakthrough in lung cancer treatment. Actually, scholars has begun their researches into immunotherapy for really a long time. Theoretically, cancer cells can be recognized as "non-self" by the host immune system though the new antigens they generated. However, there are many reported mechanisms for them to escape from the anti-tumor immunity, such as downregulation the antigen-presenting, immunoediting, induction of self-tolerance and up-regulation of immune checkpoints in the tumor micro-environment ${ }^{[10]}$. Many researches have been carried out, aiming to find an effective immunotherapy for cancer patients. At first, researchers focused on anti-cancer vaccines and nonspecific immune stimulants, which did not reveal significant effect ${ }^{[11-12]}$. Then they start to put an eye on targeting negative regulators of the immune system, which showed significant anti-tumor activity in many types of solid tumors ${ }^{[13]}$. Up to now, two major pathways have came under observation in metastatic NSCLC, corresponding to two relevant immune checkpoints. The first one is cytotoxic T-lymphocyte-associated antigen 4 (CTLA-4). CTLA-4 is a CD28 homolog which is only expressed on T cells ${ }^{[14]}$. It can bind B7 to format CTLA-4:B7 binding though competing with CD28, resulting in a negative signal to inhibit the production IL-2 and cell cycle progression so that to prevent the activation of T cell ${ }^{[15]}$. It was proved CTLA-4 blockade could enhance anti-tumor response in some preclinical studies ${ }^{[16]}$. And positive results has been achieved in clinical trials. The most representative drugs are ipilimumab and tremelimumab ${ }^{[17-19]}$. The other immune checkpoint is programmed cell death protein-1 (PD-1), which is expressed on the surface of activated T cells, B cells and natural killer cells ${ }^{[20]}$. PD-1 can recognize two known ligands, programmed cell death ligand-1 (PD-L1) and programmed cell death ligand-2 (PD-L2), to down-regulates the effectiveness of T cells. And PD-L1 was found to express on surface of various kinds of cells including the T cells. Moreover, it was also found over-expressed on tumor cells ${ }^{[21]}$. The results of preclinical studies have showed the PD-1 and PD-L1 blockade could inhibit tumor growth, which provided the evidence for clinical trials ${ }^{[22]}$. Up to now, there are many PD-1/PD-L1 inhibitors being proved their efficacy in treating NSCLC, including nivolumab $^{[23]}$, pembrolizumab ${ }^{[24]}$, and atezolizumab ${ }^{[25]}$. 
Though immunotherapy have dramatically changed the treatment patterns of NSCLC patients, not all NSCLC patients have gained the survival benefit. Also they had to face the same drug-resistance problem. Hence, the researchers begun to explore combination therapy. The first one try is the ICls combined with chemotherapy. L. Gandhi etc has demonstrated a better overall survival and progression-free survival for NSCLC patients without EGFR or ALK mutations by treating pembrolizumab combined platinum-based drug as first line therapy ${ }^{[26]}$. This synergistic effect was also proved in some other studies ${ }^{[27-28]}$, which provided a new treatment strategy for NSCLC patients. For the different timing working on T cells and different locations between CTLA-4 and PD-1, researchers also tried a combination treatment with CTLA-4 and PD-1 inhibitors, which is a research hotspot recently. Some studies have showed nivolumab plus ipilimumab could result in a longer overall survival than chemotherapy in patients with advanced or recurrent NSCLC ${ }^{[29]}$. Moreover, researches on the ICls combination with EGFR-TKIs or antiangiogenic agents are ongoing too ${ }^{[30-32]}$

Besides, researchers are also studying on the combination of ICls with radiotherapy (RT) for its immunomodulatory effect has been found. And many researches have being carried out to explore the potential mechanisms, which as follows: inducing immune responses ${ }^{[33]}$, killing the immunogenic cells which will release tumor antigens ${ }^{[34]}$, activating the "stimulator of interferon genes"to generate an adequate immune response ${ }^{[35]}$, improving the ability of tumor cell recognition [36]. Moreover, the more pronounced tumor regression by combination of ICls and RT has been proved by several preclinical studies ${ }^{[37-39]}$, which strengthens the hypothesis that RT can be an immunomodulatory in combination with immunotherapy. The results of Antonia SJ etc's study has showed that durvalumab, as consolidation immunotherapy after concurrent platinum-based chemoradiation for unresectable stage III NSCLC patients, resulted in significantly longer overall survival than placebo ${ }^{[40]}$. But for advanced NSCLC patients, there is still controversy about the efficacy, safety and timing of combination of RT with ICls. Therefore, our meta-analysis is trying to explore these questions.

\section{Patents And Method}

\section{Search strategy and study selection}

We used free-text words and MeSH terms to do the research. Two investigators (Dan Yang and LuLu Wang) independently searched for studies that published in English from PubMed, SpringerLink, Embase, Web of Science, Medline, and the Cochrane Library, using the keywords including pembrolizumab or nivolumab or atezolizumab or durvalumab or ipilimumab or PD-1 or PD-L1 or CTLA-4 or ICls, RT and non-small cell lung cancer. The last search was performed on June 1, 2020. We also performed the reviewed abstracts from major conference proceedings of a computerized search of American Society of Clinical Oncology (ASCO), the European Society of Medical Oncology (ESMO), and the American Association for Cancer Research (AACR), the European Lung Cancer Conference (ELCC) and the World Conference on Lung Cancer (WCLC). We only included the most complete and updated data of the study.

The inclusion criteria were below: (i) the study reported using ICls plus non-traditional RT in advanced lung cancer patients; (ii) any of the following measurements were investigated: response rate (RR), disease control rate (DCR), progression-free survival (PFS), and overall survival (OS); (iii) the study was a clinical trial published in English.

The following exclusion criteria were used: (i) case reports, editorials, letters, expert opinions, or reviews; (ii) studies don't publish any related data; (iii) repeated publications.

\section{Data extraction and quality assessment}

Two authors (Dan Yang and LuLu Wang) independently extracted the data. Any discrepancies were resolved by consensus. The following information was extracted from each study: first author, number of patients, randomization, trial phase, year of publication or conference presentation, clinical characteristics of enrolled patients including PD-L1 level, ICls drug, the timing and dose of RT, follow-up information, treatment outcomes including RR, DCR, PFS, OS, sixmonth PFS rate (PFSR6m), or one-year OS rate (OSR1y) and treatment-related adverse events (AEs).

The I2 statistic was used to evaluate the heterogeneity between studies. It was considerd significant heterogeneity If I2>50\%. An effect model by MantelHaenszel was used if heterogeneity was observed and subgroup analysis was performed to reduce heterogeneity ${ }^{[41]}$. Possible publication bias was detected by funnel plots. To assess the stability of combined results by excluding studies one by one, sensitivity analysis was performed. Most analyses were performed using STATA version 16.0 (Stata Corporation, College Station, TX).

\section{Quality control}

We used the Cochrane Collaboration's tool to conduct the risk of bias assessment of the random trails. We used the Newcastle Ottawa Scale (NOS) to evaluate the quality of enrolled single-arm or non-controlled studies ${ }^{[42]}$. Studies were divided into three grades: poor, modest, and high quality, according to scores ranging from 0 to 3,4 to 6 , and 7 to 9 , respectively.

\section{Results}

\section{Literature search}

The flow diagram for study inclusion is revealed in Figure 1. The preliminary literature search identified 683 abstracts, and 673 papers from databases that fulfilled our inclusion criteria. Titles and abstracts were reviewed, and 331 potential full-text articles were evaluated for eligibility. All relevant references were reviewed. Ten clinical trials were finally identified for inclusion in our study, including 7 non-randomized trials and 3 randomized trials.

Seven of these 10 studies reported the efficacy of anti-PD-1/PD-L1 antibodies plus RT in the treatment of advanced lung cancer, two investigated the efficacy of CTLA4 antibody plus RT, and one investigated the efficacy of anti-PD-1/PD-L1 antibodies plus CTLA4 antibody plus RT.

Page 3/11 


\section{Study characteristics}

Four of the studies were two-armed while the rest six studies without a control group. Most trials were phase 1/2 studies. In total, 3995 patients were enrolled in this meta-analysis. All studies were from 5 different countries, most of them were from USA; the rest were from France, Italy, Spain, and Netherlands separately. Most of the patients had a PS of $0-1$. The median follow-up ranged from 7.3 to 23.6 months.

Seven of the ten researches provided data to extract the overall response rate (ORR) while six were available to extract the DCR. Meanwhile, seven studies were available to extract the OSR1y and six studies were available to determine the PFSR6m. Details of the study characteristics are showed in Table 1.

\section{Main analysis}

We assessed the efficacy of combination regimens of ICls and RT separately according to the data they provided (Figure 2). The combined ORR and DCR was $34 \%$ ( 95\% Cl: 19\%-49\%) and $72 \%$ ( 95\% Cl: 65\%-79\%) separately. The combined PFSR6m and OSR1y values were 50.0\% (29.4\%-72.8\%) and 59.0\% (48\%-69\%), respectively. The fixed-effect model was used for DCR while the random-effect model was used for the other three analyses because of the significant heterogeneity.

Among the four two-armed studies, three studies provided the ORR and OSR1y of the control groups, while only two studies provided the DCR and PFSR6m. The combined ORR and DCR was $25 \%$ ( 95\% Cl: 16\%-34\%) and $52 \%$ ( 95\% Cl: 39\%-64\%) separately. The combined PFSR6m and OSR1y values were 35\% $(23 \%-47 \%)$ and $41.0 \%(23 \%-60 \%)$, respectively. The random-effect model was used to access the OSR1y values because of the significant heterogeneity $\left(\left(1^{2}=0.853\right)\right.$.

\section{Subgroup analyses}

To investigate possible reasons for the heterogeneity, we performed subgroup analyses according to whether the trial was two-armed, whether the trail was prospective, the different types of ICls, the different line of treatment, the different timing of RT.

Five studies were two-armed studies. But only two studies provided HR values of PFS while there studies provided HR values of OS. The combined HR and its $95 \% \mathrm{Cl}$ for PFS of the two studies was $0.64(0.46-0.90)$, with statistical significance $(\mathrm{z}=2.55, \mathrm{p}=0.01)$. This results indicates that ICls combined with RT led to a significant improvement in PFS compared with ICls alone in advanced NSCLC. Meanwhile, the combined HR and its $95 \% \mathrm{Cl}$ for OS of the three studies was $0.62(0.46-0.85)$, with statistical significance $(z=2.99, p=0.003)$. This results also indicates that ICls combined with RT led to a significant improvement in $0 S$, comparing with ICls alone in advanced NSCLC. A forest plot is shown in Figure 3.

Seven studies were prospective studies, and all these studies have provided the ORR of the combination therapy group. The combined ORR was $33.9 \% \otimes 18.6 \%-49.3 \% \varangle$ with significant heterogeneity ( $Q$ statistic, $34.75 ; I^{2}, 82.7 \% ; p=0.000$ ). A random-effect model was performed. Six studies have provided the DCR and the combined DCR was $72.4 \% \varangle 65.3 \%-79.5 \% \bigotimes$ with good heterogeneity ( $Q$ statistic, $3.24 ; I^{2}, 0.0 \% ; p=0.663$ ). A fixed-effect model was performed. Four studies have provided the PFS6m and OSR1y of the combination therapy group. The combined PFS6m and 0SR1y was 50.5\% $25.0 \%-76.0 \% \bigotimes$ and $58.9 \% \bigotimes 38.5 \%-79.4 \% \bigotimes$ respectively, with significant heterogeneity. A random-effect model was performed.

Six studies investigated the efficacy of an anti-PD-1/PD-L1 antibody combined with RT. Four studies provided the ORR and DCR of the study groups. The combined ORR was $31.5 \% \otimes 16.4 \%-46.6 \%$ with significant significant heterogeneity ( $Q$ statistic, $7.86 ; I^{2}, 61.8 \% ; p=0.049$ ). A random-effect model was performed. The combined DCR was $72.1 \%(63.3 \%-80.9 \%)$ with good significant heterogeneity (Q statistic, 3.17; $\left.I^{2}, 5.5 \% ; p=0.366\right)$. A fixed-effect model was performed. Five studies provided the PFS6m of the study groups. The combined PFS6m was $50.0 \% \otimes 20.6 \%-79.5 \%$ \&with significant significant heterogeneity (Q statistic, $\left.67.57 ; I^{2}, 94.1 \% ; p=0.000\right)$. A random-effect model was performed. Six studies provided the OS1y of the study groups. The combined OSR1y was $59.7 \% \varangle 48.1 \%-71.2 \% \rrbracket$ with significant heterogeneity (Q statistic, $20.47 ; l^{2}, 75.6 \% ; p=0.001$ ). A random-effect model was performed. Only two studies investigated the efficacy of an anti-CTLA4 antibody combined with RT and only provided the ORR of the combination treatment group. The combined ORR was $19.5 \%$ (9.1\%-29.8\%) with good significant heterogeneity (Q statistic, $0.26 ; I^{2}, 0.0 \% ; p=0.612$ ). A fixed-effect model was performed.

We divided these trails into two subgroups according to the timing of RT. One is the concurrent therapy group, in which patients received RT at the first dose of ICls or after the first dose. The other one is the sequential group, in which patients received the first dose of ICls after the completion of RT. There are five studies in the concurrent therapy group. All five studies provided the ORR and the combined ORR was $21.3 \% \otimes 13.9 \%-28.6 \% \rrbracket$ with good heterogeneity (Q statistic, $\left.2.37 ; I^{2}, 0.0 \% ; p=0.669\right)$. A fixed-effect model was performed. Four studies provide the DCR and the combined DCR was $71.8 \% \llbracket 62.0 \%-81.6 \% \bigotimes$ with good heterogeneity ( $Q$ statistic, 3.18; $\mathrm{I}^{2}, 5.7 \% ; \mathrm{p}=0.365$ ). A fixed-effect model was performed. Three studies have provided the PFS6m and OSR1y of the concurrent therapy group. The combined PFS6m and OSR1y were 58.9\% $26.2 \%-91.7 \% \llbracket$ and $59.7 \% \varangle 29.7 \%-89.8 \%$ respectively, with significant heterogeneity. A randomeffect model was performed. Meanwhile, only two studies investigated the efficacy of sequential therapy. According to the data provided, we can only do the combination of PFS6m and OS1y. The results were $55.2 \% \varangle 41.6 \%-68.8 \% \llbracket$ and $55.7 \% \varangle 42.1 \%-69.3 \% \bigotimes$ respectively, with good heterogeneity (Q statistic, $0.21 ; I^{2}$, $0.0 \% ; p=0.649$ ). A fixed-effect model was performed.

Among all the enrolled studies, the total dose and fractionation of RT significant differed. We defined a low-dose group when RT dose <45Gy, and a high-dose group if RT dose $\geqq 45 \mathrm{~Gy}$. Three studies investigated the efficacy of combination with low-dose RT. All three studies provided the ORR and the DCR of the study groups. The combined ORR was 30.2\% $\$ 10.7 \%-49.6 \% \rrbracket$ with significant heterogeneity ( $Q$ statistic, $7.47 ; 12,73.2 \% ; p=0.024$ ). A random-effect model was performed. The combined DCR was $69.7 \% \varangle 59.8 \%-79.6 \% \bigotimes$ with good heterogeneity ( $Q$ statistic, $2.12 ; 12,5.9 \% ; p=0.346$ ). A fixed-effect model was performed. Two studies have provided the PFS6m and OSR1y of the low-dose RT therapy group. The combined PFS6m was $45.9 \% \otimes 27.0 \%-64.8 \% \rrbracket$ with modestly significant heterogeneity ( $Q$ statistic, 1.6; I2, 37.3\%; $p=0.207$ ). A random-effect model was performed. The combined OS1y was $51.6 \% \varangle 37.5 \%-65.6 \% \varangle$ with good

Page $4 / 11$ 
heterogeneity ( $Q$ statistic, $0.61 ; 12,0.0 \% ; p=0.436)$. A fixed-effect model was performed. Three studies investigated the efficacy of combination with high-dose RT. All three studies provided the ORR of the study groups. The combined ORR was $22.3 \% \otimes 12.8 \%-31.8 \% \varangle$ with good heterogeneity (Q statistic, 2.02 ; I2, $1.2 \%$; $p$ $=0.363$ ). A fixed-effect model was performed. Two studies provided the DCR of the study groups. The combined DCR was $76.6 \% \bigotimes 62.2 \%-90.9 \% \bigotimes$ with good heterogeneity ( $Q$ statistic, $0.46 ; 12,0.0 \% ; p=0.498$ ). A fixed-effect model was performed. Two studies have provided the PFS6m and OSR1y. The combined PFS6m and OS1y were 71.1\% $37.9 \%-104.4 \% \bigotimes$ and $67.4 \% \varangle 29.2 \%-105.6 \% \bigotimes$ separately, with significant heterogeneity. A random-effect model was performed.

Patients in three studies received the combination therapy as first-line therapy. Two studies provided the ORR and the DCR of the study groups. The combined ORR was $53.8 \% \varangle 23.6 \%-84.1 \% \varangle$ with significant heterogeneity (Q statistic, 4.62; I2, 78.4\%; $p=0.032$ ). A random-effect model was performed. The combined DCR was $76.5 \% \varangle 65.0 \%-88.1 \% \varangle$ with good heterogeneity ( $Q$ statistic, $0.32 ; 12,0.0 \% ; p=0.569$ ). A fixed-effect model was performed. Patients in other six studies received the combination therapy as at least second-line therapy. Five studies provided the ORR of the study groups. The combined ORR was

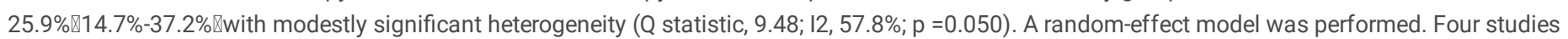
provided the DCR of the study groups. The combined DCR was $69.9 \% \varangle 60.9 \%-78.9 \% \varangle$ with good heterogeneity $(\mathrm{Q}$ statistic, $2.14 ; \mathrm{I} 2,0.0 \% ; \mathrm{p}=0.545)$. A fixedeffect model was performed. Four studies have provided the PFS6m and OSR1y of the combination group. The combined PFS6m and OS1y were $51.1 \% \varangle 40.3 \%-61.9 \% \rrbracket$ and $51.8 \% \varangle 40.9 \%-62.7 \%$ respectively, both with good heterogeneity. A fixed-effect model was performed.

\section{Tolerability}

Generally speaking, sides' effects were tolerable. The most commonly reported treatment-related AEs of 3/4 grade for the combination of ICls and RT were endocrinological toxicities, asthenia, hematological toxicities, pneumonitis, and dermatological toxicities.

\section{Sensitivity analysis and publication bias}

We performed sensitivity analysis to detect the robustness of the combined outcomes. Omitting any single trial did not significantly alter the results. We also used a funnel plot to observe the potential of publication bias for the ORR, DCR, PFSR6m and OSR1y of the ICls plus RT therapy in NSCLC patients. The possible reason might be the limit number of trials.

\section{Discussion}

In recent years, several ICls medicines, including anti-PD-1/PD-L1 antibody and anti-CTLA4 antibody, have been improved good efficacy in NSCLC patients ${ }^{[18-}$ 19, 23-25]. In order to get better efficacy, combination therapy have been proposed, such as ICls plus chemotherapy, ICls plus target therapy, anti-PD-1/PD-L1 antibody plus anti-CTLA4 antibody and ICls plus RT. For patients with unresectable stage III NSCLC, durvalumab has showed its advantage to improve their OS and PFS, as maintenance therapy after concurrent chemoradiation ${ }^{[40]}$. However, for patients with advanced NSCLC, the efficacy of ICls plus RT is still uncertain. So we performed this meta-analysis to investigate potential therapy strategies for advanced NSCLC patients.

ICls were an inspiring breakthrough in cancer therapy in recent years. The ORR of single therapy ranged from $19.4-20 \%$, and the 0S1y ranged from $42 \%-47.9 \%$ $[19,24-25]$. From our results, the combined ORR of ICls plus RT is $34 \%$, and the OS1y is $59 \%$, which revealed an impressive efficacy of the combination therapy with tolerable side effects. This result was consistent with many preclinical studies, which showed RT could cause the immunomodulatory effect to prove the more pronounced tumor regression by combination with ICls ${ }^{[33-39]}$. Moreover, our results showed a significant improvement in both PFS (\%95 Cl: $0.46-0.90 \mathrm{p}$ $=0.01)$ ) and OS (\%95 Cl: $0.46-0.85 \mathrm{p}=0.003)$ for the combination therapy of ICls plus RT comparing with the singe ICls therapy, which revealed the combination therapy might be a better therapy strategy for advanced NSCLC. But in our study there are only two two-armed trails providing the HR values of PFS and three trails providing the HR values of OS. More prospective trials are ongoing now. Therefore, further study is needed to verify this outcome.

As we said before, there are two types of ICls widely being used in clinical treatment for NSCLC. They are anti-PD-1/PD-L1 antibody and anti-CTLA4 antibody. From previous report, the ORR for anti-PD-1/PD-L1 antibody and anti-CTLA4 antibody single treatment were $20 \%$ and $4.8 \%$ respectively. In our study, the results showed that combined ORR were $31.5 \%$ and $19.5 \%$ respectively, which revealed that the combination with RT could sharply improve the efficacy for both antiPD-1/PD-L1 antibody and anti-CTLA4 antibody. But the efficacy was different between them, the ORR of anti-PD-1/PD-L1 antibody plus RT was higher than anti-CTLA4 antibody plus RT, which was accordant with the results when they were used as single therapy treating patients with melanoma ${ }^{[53]}$. This might because though both anti-PD-1/PD-L1 antibody and anti-CTLA4 antibody showed the efficacy on treating cancers, but for the difference of their responsible signaling mechanisms and their ligands' location, they also differed on the efficacy on anti-tumors. This theory also provided another way of combination therapy of anti-PD-1/PD-L1 antibody plus anti-CTLA4 antibody plus RT. The only included trial (NCT03223155), exploring the efficacy of anti-PD-1/PD-L1 antibody plus anti-CTLA4 antibody plus RT, is a prospective trial, which is still ongoing. The preliminary result showed a really good result of ORR (68\%), which is much higher than double medicine combination. We will pay close attention to the final result and will also concern about the tolerance of patients.

As for the timing of RT when combined with ICls, the best strategy is still uncertain. Like we said before, inducing immune responses is one potential mechanisms for RT to enhance the efficacy of ICls. This was reported to be achieved by the increase of cell surface-MHC class I expression, which can be test just $18 \mathrm{~h}$ after RT ${ }^{[36]}$. On the other hand, it was also observed that the PD-L1 expression was significantly increased after RT, which might reveal a worse response to RT in NSCLC ${ }^{[38]}$. Through down regulating PD-L1 expression, the percentage of apoptotic cells was significantly increased after radiation than that in the cells without radiation. So that the radiation resistance was alleviated. Moreover, the combination of anti-PD-1/PD-L1 antibody and RT could reduce the accumulation of MDSCs, which has also been demonstrated to be relative with radiation resistance ${ }^{[54]}$. Therefore, we can conclude that the anti-PD-1/PDL1 antibody may also improve the efficacy of RT by blockade of PD-L1. Taken together, it seems that concurrent ICls and RT would have better efficacy than sequential therapy, because ICls and RT can enhance each other's effectiveness. But our results did not show any significant difference on the combined PFS6m and 0S1y between these two types of combination strategies. However, the number of enrolled studies for sequential therapy is limited, which might 
affect the result. And in the PEMBRO-RT trial, which was a random prospective study for sequential therapy, the first course of ICls was given within 7 days after completion of RT. Though it was included in the sequential therapy group, there was not a long interval time between ICls and RT. After all, the best timing for the combination of ICls and RT still need more prospective study for further confirm.

Another uncertain problem is the dose of RT when combined with ICls. The result of Eric A. Refits' study showed that the increase of cell surface-MHC class I expression after radiation was dose dependent ${ }^{[36]}$. Moreover, the increase of PD-L1 expression after radiation was also reported to be dose relative ${ }^{[38]}$. We hypothesized the dose of RT might also affect the efficacy of combination therapy. Since, we analyzed the efficacy of low-dose RT group and high-dose RT group separately. The results showed that the low-dose group had a better ORR. But the PFS6m (71.1\% vs $45.9 \%)$ and OS1y (67.4\% vs $51.6 \%)$ of high-dose group were much higher, which seemed to confirm our hypothesis. For the high-dose RT might accompany with higher incidence of side effects, the best total dose remains uncertain. The dose fractionation might be another factor to affect the results. But we could not analyze the efficacy of different ways of dose fractionation, for it differed in different studies and even in the same study it was not uniform. So more prospective studies to explore the dose and the fraction strategy are needed.

In our studies, most trials investigated ICls plus RT as subsequent therapy in advanced NSCLC. The results showed the combined ORR was $25.9 \%$, PFS6m was $51.1 \%$ and OS1y was $51.8 \%$, while they were reported $18-20 \%, 28.1-35.5 \%$ and $42-51 \%$ separately for single ICls treatment as subsequent therapy $[23,24,55]$. It seems that the combined therapy lead to a better ORR and PFS, but the similar OS. In our study, NCT02444741 is the only prospective study investigating ICls plus RT as first-line treatment. Their results did not show significant difference between two groups not only in RR $(22 \%$ vs $25 \%, P=1.00)$, but also in PFS (10.9 vs 8.4 months, $p=0.83$ ). As for the non-traditional fractionation group, which was more concerned in our study, it showed a much better ORR ( $38 \%)$ and a longer PFS (21.1 months). For the elementary data of this trial was just published in the conference report, more data was expected.

Although just few studies were enrolled in our meta-analysis, this is the first meta-analysis to evaluate the efficacy and safety of the combination of ICls plus RT as treatment strategies in advanced NSCLC. To minimize the influence of heterogeneity, we used the random-effects model and performed exploratory subgroup analyses based on whether the trial was two-armed, whether the trail was prospective, the different types of ICls, the different lines of treatment and the different timing of RT.

However, our meta-analysis had several limitations. Firstly, most of the studies just had small sample sizes because studies focusing on ICls plus RT for advanced NSCLC are not much. Secondly, most trails only involved single-arm studies without randomization. Thirdly, most trails did not define the exact the dose fractionation and timing of RT. Fourthly, we widely use the random-effects model in our analysis, which may increase the effects of small samples with poor quality studies. Finally a potential impact of publication bias and selection bias might existed. These limitations may have affected the final results.

In our study, the data are insufficient, but various trials are still ongoing. Some more single-armed trials are investigating the efficacy and safety of ICls plus RT, including NCT03557411, NCT03812549 and NCT03705806. There are also more trials are comparing the efficacy between ICls plus RT and ICls, including NCT03774732, NCT03867175 and NCT02658097. The antitumor effect of PD-1/PD-L1 antibody plus CTLA4 antibody on NSCLC was proved. The efficacy of double checkpoint inhibitors in lung cancer plus RT is being evaluated in one ongoing trial (NCT03275597). Moreover the combination of ICls plus RT is also evaluated as neoadjuvant therapy in one ongoing trail (NCT04245514). And this trial also is investigating the best RT fractionation for combination therapy. These results will elucidate the efficacy of ICIs plus RT.

\section{Conclusions}

Our meta-analysis suggests that combination treatment with ICls plus RT is an effective and tolerable option as a treatment for advanced NSCLC. Further prospective, multicenter, randomized control trials are urgently needed to validate our results. The location, dose and fractionation of RT should be considered in the design of future studies. The costs of these strategies together with patients'quality of life should also be carefully evaluated.

\section{Abbreviations}

NSCLC, non-small-cell lung cancer; ORR, objective response rate;DCR, disease control rate; PFS, progression-free survival; OS, overall survival; PFSR6m, sixmonth progression-free survival rates; OSR1y, one-year overall survival rate; HR, hazards ratio; EGFR, epidermal growth factor receptor; TKI, Tyrosine Kinase Inhibitor; ALK, anaplastic lymphoma kinase;POS-1, ROS proto-oncogene 1; ICls, immune checkpoint inhibitors; CTLA-4, cytotoxic T-lymphocyte-associated antigen 4; PD-1, programmed cell death protein-1; PD-L1, programmed cell death ligand-1; PD-L2, programmed cell death ligand-2; RT, radiotherapy; AEs, adverse events;

\section{Declarations}

\section{Disclosure}

No authors report any conflict of interest.

\section{Funding}

Funded by Chongqing Scientific Research Institutes performance incentive guide special youth project(cstc2019jxjl0051). 


\section{Acknowledgment}

The Chongqing Scientific Research Institutes performance incentive guide special youth project supported this study (cstc2019jxjl0051). We thank all of the participants. We wish to thank all of the doctors who assisted us with this study.

\section{References}

1. Siegel RL., Miller KD., Jemal A. Cancer statistics, 2018. CA Cancer J Clin 2018; 68(1): 7-30.

2. Chen W, Zheng R, Baade PD, et al. Cancer statistics in China, 2015. CA Cancer J 2016; 66(2):115-132. doi:10.3322/caac.21338.

3. Rami PR. New TNM classification for lung cancer. Arch Bronconeumol 2009; 45: 159-161.

4. Novello S, Barlesi F, Califano R, et al. Metastatic non-small-cell lung cancer: ESMO Clinical Practice Guidelines for diagnosis, treatment and follow-up. Annals of Oncology 2016; 27:v1-v27.

5. Chemotherapy in non-small cell lung cancer: a meta-analysis using updated data on individual patients from 52 randomised clinical trials. Non-small Cell Lung Cancer Collaborative Group. BMJ. 1995; 311(7010):899-909.

6. Shaw AT, Kim DW, Nakagawa K, et al. Crizotinib versus chemotherapy in advanced ALK-positive lung cancer. N Engl J Med 2013; 368: 2385-94.

7. Shaw AT, Ou SH, Bang YJ, et al. Crizotinib in ROS1-rearranged non-small-cell lung N Engl J Med 2014; 371: $1963-71$.

8. Yang JC, Sequist LV, Geater SL, et al. Clinical activity of afatinib in patients with advanced non-small-cell lung cancer harbouring uncommon EGFR mutations: a combined post-hoc analysis of LUXLung 2, LUX-Lung 3, and LUX-Lung 6. Lancet Oncol 2015; 16: 830-38.

9. Shi Y, Au JS, Thongprasert S, et al. A Prospective, Molecular Epidemiology Study of EGFR Mutations in Asian Patients with Advanced Non-Small-Cell Lung Cancer of Adenocarcinoma Histology (PIONEER). J Thorac Oncol. 2014; 9:154-162.

10. Brahmer JR, Pardoll DM. Immune checkpoint inhibitors: Making immunotherapy a reality for the treatment of lung cancer. Cancer Immunol Res 2013 ; 1:85-91.

11. Atkins MB, Lotze MT, and Dutcher JP et al. High-dose recombinant interleukin 2 therapy for patients with metastatic melanoma: Analysis of 270 patients treated between 1985 and 1993. J Clin Oncol 1999; 17:2105-211.

12. Medical Research Council Renal Cancer Collaborators. Interferon-alpha and survival in metastatic renal carcinoma: Early results of a randomised controlled Lancet 1999; 353:14-17.

13. Topalian SL, Hodi FS, Brahmer JR et al. Safety, activity, and immune correlates of anti-PD-1 antibody in cancer. N Engl J Med 2012; 366:2443-2454.

14. Brunet JF, Denizot F, Luciani MF et al. A new member of the immunoglobulin superfamily-CTLA-4. Nature 1987; 328:267-270.

15. Krummel MF, Allison JP. CTLA-4 engagement inhibits IL-2 accumulation and cell cycle progression upon activation of resting T cells. J Exp Med. 1996; 183:2533-2540.

16. Leach DR, Krummel MF, Allison JP. Enhancement of antitumor immunity by CTLA-4 blockade. Science 1996; 271:1734-1736.

17. Hodi FS, O'Day SJ, McDermott DF et al. Improved survival with ipilimumab in patients with metastatic melanoma. N Engl J Med 2010; 363: 711-723.

18. Lynch TJ, Bondarenko I, Luft A et al. Ipilimumab in combination with paclitaxel and carboplatin as first-line treatment in stage IIIB/IV non-small-cell lung cancer: Results from a randomized, double blind, multicenter phase II study. J Clin Oncol 2012; 30:2046-2054.

19. Zatloukal P, Heo D, Park K et al. Randomized phase II clinical trial comparing tremelimumab (CP-675,206) with best supportive care (BSC) following firstline platinum-based therapy in patients (pts) with advanced non-small cell lung cancer (NSCLC). J Clin Oncol 2009; 27(suppl 15):8071a.

20. Agata Y, Kawasaki A, Nishimura H et al. Expression of the PD-1 antigen on the surface of stimulated mouse T and B lymphocytes. Int Immunol 1996; 8: 765-772.

21. Engagement of the PD-1 immunoinhibitory receptor by a novel B7 family member leads to negative regulation of lymphocyte activation. J Exp Med 2000; 192: 1027-1034.

22. Hirano F, Kaneko K, Tamura H, et al. Blockade of B7-H1 and PD-1 by monoclonal antibodies potentiates cancer therapeutic immunity. Cancer Res. 2005;65:1089-1 096.

23. Brahmer, K.L. Reckamp, P. Baas, L. Crino, W.E. Eberhardt, E. Poddubskaya,S. Antonia, A. Pluzanski, E.E. Vokes, E. Holgado, D. Waterhouse, N. Ready, J. Gainor, O. Aren Frontera, L. Havel, M. Steins, M.C. Garassino, J.G. Aerts, M. Domine, L. Paz-Ares, M. Reck, C. Baudelet, C.T. Harbison, B. Lestini, D.R. Spigel, Nivolumab versus docetaxel in advanced squamous-cell non-small-cell lung cancer, N. Engl. J. Med. 373 (2015) 123-135.

24. B. Garon, M.D. Hellmann, N.A. Rizvi, E. Carcereny, N.B. Leighl, M.J. Ahn, J.P. Eder, A.S. Balmanoukian, C. Aggarwal, L. Horn, A. Patnaik, M. Gubens, S.S. Ramalingam, E. Felip, J.W. Goldman, C. Scalzo, E. Jensen, D.A. Kush, R. Hui, Five-year overall survival for patients with advanced nonsmall-cell lung cancer treated with pembrolizumab: results from the phase I KEYNOTE-001 study, J. Clin. Oncol. 37 (2019) 2518-2527.

25. Fehrenbacher, A. Spira, M. Ballinger, M. Kowanetz, J. Vansteenkiste, J. Mazieres, K. Park, D. Smith, A. Artal-Cortes, C. Lewanski, F. Braiteh, D. Waterkamp, P. He, W. Zou, D.S. Chen, J. Yi, A. Sandler, A. Rittmeyer, P.S. Group, Atezolizumab versus docetaxel for patients with previously treated non-small-cell lung cancer (POPLAR): a multicentre, open-label, phase 2 randomised controlled trial, Lancet 387 (2016) 1837-1846.

26. Gandhi L, Rodriguez-Abreu D, Gadgeel S, et al. Pembrolizumab plus chemotherapy in metastatic non-small-cell lung cancer. N Engl J Med 2018; 378:20782092.

27. Paz-Ares L. Luft A, Vincente D, et al. Pembrolizumab plus chemotherapy for squamous non-smallcell lung cancer. N Engl J Med 2018; $379: 2040-2051$.

28. West H, McCleod M, Hussein M, et al. West H, McCleod M, Hussein M, et al. Atezolizumab in combination with carboplatin plus nab-paclitaxel chemotherapy compared with chemotherapy alone as first-line treatment for metastatic non-squamous non-small-cell lung cancer (IMpower130): a 
multicentre, randomised, open-label, phase 3 trial. Lancet Oncol. 2019; 20:924-937.

29. Hellmann MD, Paz-Ares L, Bernabe Caro, R, et al. Nivolumab plus ipilimumab in advanced nonsmall-cell lung cancer. N Eng J Med 2019; 381:2020-2031.

30. Ma BBY, Cervantes A, Huseni M A. Preliminary safety and clinical activity of erlotinib plus atezolizumab from a phase Ib study in advanced NSCLC. Ann Oncol, 2016, 27(9Suppl): mdw594.005.

31. Antonia SJ, Brahmer JR, Gettinger S, et al. Nivolumab (Anti-PD-1; BMS -936558, ONO- 4538) in combination w ith platinum-based doublet Chemotherapy (PT-DC) in advanced non-small cell lung cancer (NSCLC): Metastatic non-small cell lung Int J Radiat Oncol Biol, 2014, 90 (5): S2.

32. Rizvi N A, Antonia S J, Shepherd F A, et al. Nivolumab (Anti-PD-1; BMS-936558, ONO-4538) Maintenance as Monotherapy or in Combination With Bevacizumab (BEV) for Non-Small Cell Lung Cancer (NSCLC) Previously Treated With Chemotherapy: Metastatic Non-Small Cell Lung Cancer[J]. International Journal of Radiation Oncology• Biology• Physics, 2014, 90(5): S32.

33. Schaue, W.H. McBride, T lymphocytes and normal tissue responses to radiation, Front. Oncol. 2 (2012) 119.

34. B. Sharabi, et al., Radiation and checkpoint blockade immunotherapy: radiosensitisation and potential mechanisms of synergy, Lancet Oncol. 16 (13) (2015) e498-509.

35. Deng, et al., STING-Dependent Cytosolic DNA Sensing Promotes RadiationInduced Type I Interferon-Dependent Antitumor Immunity in Immunogenic Tumors, Immunity 41 (5) (2014) 843-852.

36. A. Reits, et al., Radiation modulates the peptide repertoire, enhances MHC class I expression, and induces successful antitumor immunotherapy, J. Exp. Med. 203 (5) (2006) 1259-1271.

37. Deng, et al., Irradiation and anti-PD-L1 treatment synergistically promote anti-tumor immunity in mice, J. Clin. Invest. 124 (2) (2014) $687-695$.

38. Gong, et al., Combined radiotherapy and Anti-PD-L1 antibody synergistically enhances antitumor effect in non-small cell lung Cancer, J. Thorac. Oncol. 12 (7) (2017) 1085-1097.

39. J. Dovedi, et al., Acquired resistance to fractionated radiotherapy can be overcome by concurrent PD-L1 blockade, Cancer Res. 74 (19) (2014) $5458-5468$. 40. Antonia SJ, Villegas A, Daniel D, et al. Overall survival with durvalumab after chemoradiotherapy in stage III NSCLC. N Engl J Med 2018; 379:2342-2550.

41. Higgins JPGS, editor. Cochrane collaboration. Cochrane handbook for systematic reviews of Interventions. West Sussex: Wiley; 2008.

42. Lo CK, Mertz D, Loeb M. Newcastle-Ottawa Scale: comparing reviewers' to authors' assessments. BMC Med Res Methodol 2014; 14:45.

43. Tang C, de Groot P, Hess K, et al. Phase 1 study of pembrolizumab and stereotactic or hypofractionated radiation for metastatic non-small cell lung cancer[J]. International Journal of Radiation Oncology• Biology• Physics, 2017, 99(2): S160-S161.

44. Qin A, Rengan R, Lee S, et al. A pilot study of Atezolizumab plus hypofractionated image-guided radiotherapy for the treatment of advanced non-small cell lung cancer [J]. International Journal of Radiation Oncology* Biology* Physics, 2019.

45. Lesueur P, Escande A, Thariat J, et al. Safety of combined PD-1 pathway inhibition and radiation therapy for non-small-cell lung cancer: A multicentric retrospective study from the GFPC [J]. Cancer medicine, 2018, 7(11): 5505-5513.

46. Fiorica F, Belluomini L, Stefanelli A, et al. Immune Checkpoint Inhibitor Nivolumab and Radiotherapy in Pretreated Lung Cancer Patients[J]. American journal of clinical oncology, 2018, 41(11): 1101-1105.

47. Foster C C, Sher D J, Rusthoven C G, et al. Overall survival according to immunotherapy and radiation treatment for metastatic non-small-cell lung cancer: A National Cancer Database analysis [J]. Radiation Oncology, 2019, 14(1): 18.

48. Moreno V, Gil-Martin M, Johnson M L, et al. 72P Cemiplimab, a human monoclonal anti-PD-1, plus radiotherapy (RT) in advanced non-small cell lung cancer (NSCLC): Results from a phase I expansion cohort (EC 2) [J]. Annals of Oncology, 2018, 29(suppl_10): mdy487. 003.

49. Moreno V, Calvo E, Garcia M E O, et al. Tolerability and antitumor activity of cemiplimab, a human monoclonal anti-PD-1, in patients with non-small cell lung cancer (NSCLC): Interim data from a phase I dose escalation and NSCLC expansion cohort [J]. Journal of Clinical Oncology, 2019, 37(8_suppl): 116116.

50. Moreno V, Gil-Martin M, Johnson M, et al. MA04. 01 Cemiplimab, a Human Monoclonal Anti-PD-1, Alone or in Combination with Radiotherapy: Phase 1 NSCLC Expansion Cohorts [J]. Journal of Thoracic Oncology, 2018, 13(10): S366.

51. Theelen W S M E, Peulen H M U, and Lalezari F, et al. Effect of pembrolizumab after stereotactic body radiotherapy vs pembrolizumab alone on tumor response in patients with advanced non-small cell lung cancer: results of the PEMBRO-RT phase 2 randomized clinical trial [J]. JAMA oncology, 2019, 5(9): 1276-1282.

52. Tang C, Welsh J W, and De Groot P, et al. Ipilimumab with stereotactic ablative radiation therapy: phase I results and immunologic correlates from peripheral T cells [J]. Clinical Cancer Research, 2017, 23(6): 1388-1396.

53. Farolfi A, Ridolfi L, Guidoboni M, et al. Ipilimumab in advanced melanoma: reports of long-lasting responses. Melanoma Res.2012; 22:263-270.

54. Tadmor T, Attias D, Polliack A. Myeloid-derived suppressor cells-their role in haemato-oncological malignancies and other cancers and possible implications for therapy. Br J Haematol. 2011; 153:557-567.

55. Borghaei H, Paz-Ares L, Horn L, et al. Nivolumab versus docetaxel in advanced nonsquamous non-small-cell lung cancer [J]. New England Journal of Medicine, 2015, 373(17): 1627-1639.

\section{Tables}




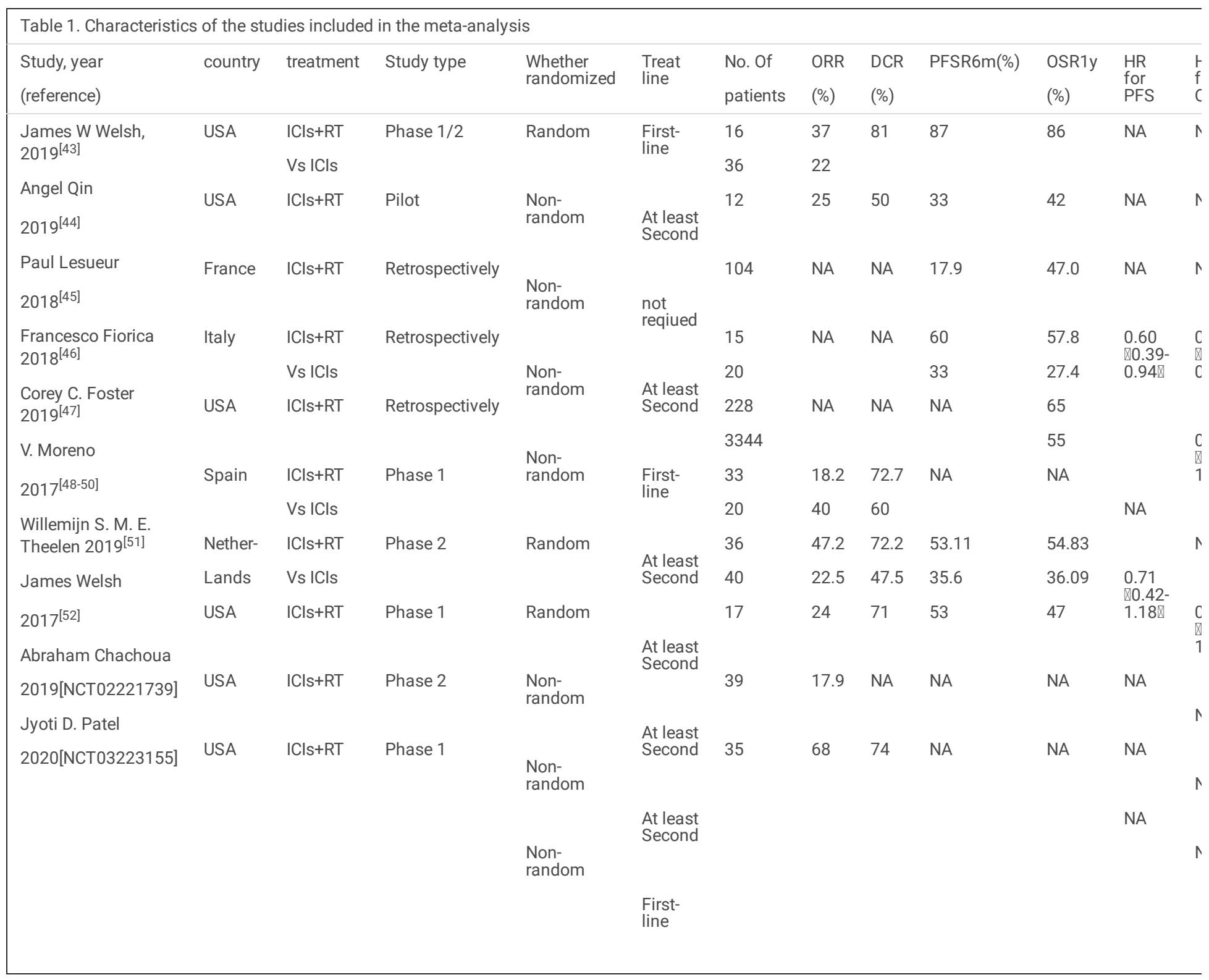




\begin{tabular}{|c|c|c|c|c|}
\hline Analyses & $\begin{array}{l}\text { Pooled ORR, model, Number } \\
\text { of studies }\end{array}$ & $\begin{array}{l}\text { Pooled DCR, model, Number } \\
\text { of studies }\end{array}$ & $\begin{array}{l}\text { Pooled PFSR6m, model, } \\
\text { Number of studies }\end{array}$ & $\begin{array}{l}\text { Pooled OSR1y, model, } \\
\text { Number of studies }\end{array}$ \\
\hline \multirow{2}{*}{$\begin{array}{l}\text { Anti-PD-1/PD-L1 } \\
\text { antibody +RT }\end{array}$} & $31.5 \% \triangle 16.4 \%-46.6 \% \square$ & $72.1 \% \varangle 63.3 \%-80.9 \% \rrbracket$ & $50.0 \% \rrbracket 20.6 \%-79.5 \% \rrbracket$ & \multirow{2}{*}{$\begin{array}{l}59.7 \% \varangle 48.1 \%-71.2 \% \bigotimes \text { Random, } \\
6\end{array}$} \\
\hline & Random, 4 & Fixed, 4 & Random, 5 & \\
\hline \multirow{2}{*}{$\begin{array}{l}\text { Anti-CTLA4 } \\
\text { antibody+RT }\end{array}$} & $19.5 \%(9.1 \%-29.8 \%)$ & NA & NA & \multirow[t]{2}{*}{ NA } \\
\hline & Fixed, 2 & & & \\
\hline \multirow[t]{2}{*}{ Prospective, ICls+RT } & $33.9 \% \varangle 18.6 \%-49.3 \% \rrbracket$ & $72.4 \% \llbracket 65.3 \%-79.5 \% \rrbracket$ & $56.9 \% \varangle 33.9 \%-79.9 \% \bowtie$ Random, 4 & $58.9 \% \rrbracket 38.5 \%-79.4 \% \rrbracket$ \\
\hline & Random, 7 & Fixed, 6 & & Random, 4 \\
\hline \multirow[t]{2}{*}{ concurrent therapy } & $21.3 \% \triangle 13.9 \%-28.6 \% \square$ & $71.8 \% \varangle 62.0 \%-81.6 \% \square$ & $58.9 \% \rrbracket 26.2 \%-91.7 \% \rrbracket$ & $59.7 \% \otimes 29.7 \%-89.8 \% \rrbracket$ \\
\hline & Fixed, 5 & Fixed, 4 & Random, 3 & Random, 3 \\
\hline \multirow[t]{2}{*}{ sequential therapy } & NA & NA & $55.2 \% \otimes 41.6 \%-68.8 \% \rrbracket$ & $55.7 \% \otimes 42.1 \%-69.3 \% \bigotimes$ \\
\hline & & & Fixed, 2 & Fixed, 2 \\
\hline \multirow[t]{2}{*}{ Low dose RT } & $30.2 \% \otimes 10.7 \%-49.6 \% \rrbracket$ & $69.7 \% \varangle 59.8 \%-79.6 \% \rrbracket$ & $45.9 \% \rrbracket 27.0 \%-64.8 \% \rrbracket$ & $51.6 \% \bigotimes 37.5 \%-65.6 \% \rrbracket$ \\
\hline & Random, 3 & Fixed, 3 & Random, 2 & Fixed, 2 \\
\hline \multirow[t]{2}{*}{ High dose RT } & $22.3 \% \triangle 12.8 \%-31.8 \% \rrbracket$ & $76.6 \% \varangle 62.2 \%-90.9 \% \rrbracket$ & $71.1 \% \llbracket 37.9 \%-104.4 \% \rrbracket$ & $67.4 \% \bowtie 29.2 \%-105.6 \% \rrbracket$ \\
\hline & Fixed, 3 & Fixed, 2 & Random, 2 & Random, 2 \\
\hline \multirow[t]{2}{*}{ First-line group } & $53.8 \% \varangle 23.6 \%-84.1 \% \rrbracket$ & $6.5 \% \varangle 65.0 \%-88.1 \% \square$ & NA & NA \\
\hline & Random, 2 & Fixed, 2 & & \\
\hline \multirow[t]{2}{*}{ At least second-line } & $25.9 \% \varangle 14.7 \%-37.2 \% \square$ & $69.9 \% \llbracket 60.9 \%-78.9 \% \rrbracket$ & $51.1 \% \varangle 40.3 \%-61.9 \% \rrbracket$ & $51.8 \% \otimes 40.9 \%-62.7 \% \rrbracket$ \\
\hline & Random, 5 & Fixed, 4 & Fixed, 4 & Fixed, 4 \\
\hline
\end{tabular}

\section{Figures}

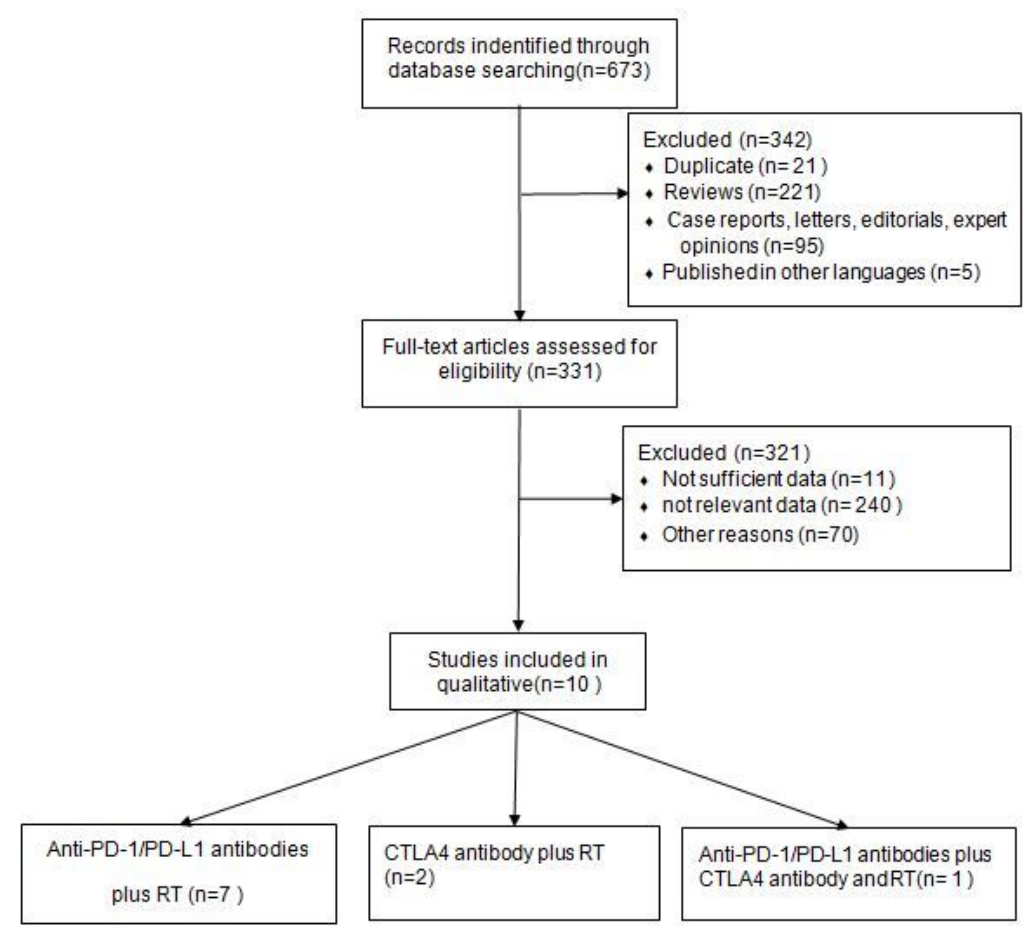

\section{Figure 1}

Flowchart of the study selection procedure 

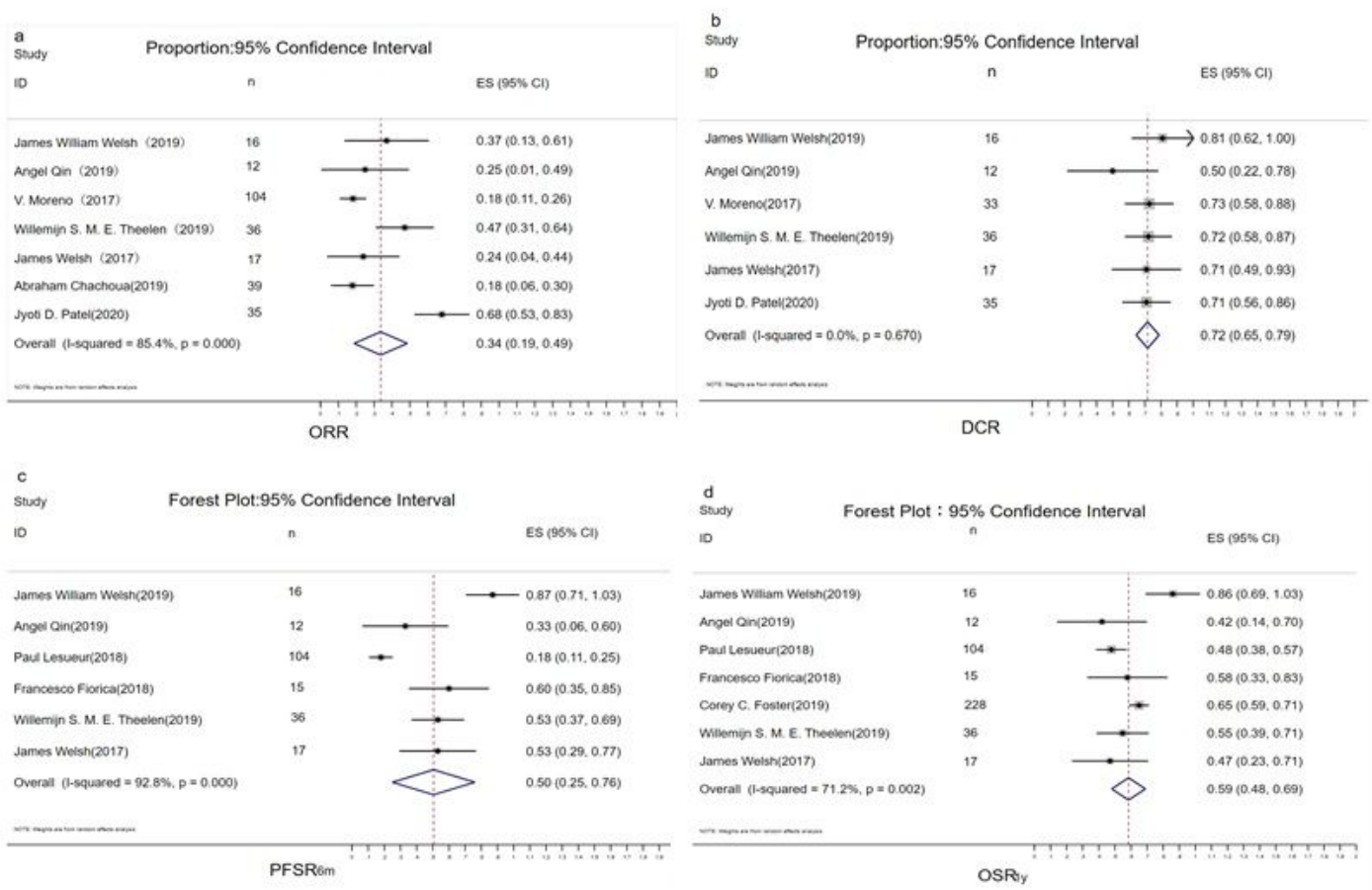

Figure 2

Forest plot of the combined ORR, DCR, PFSR6m, or OSR1y of ICls combined with radiotherapy as treatment for advanced lung cancer. Among the ten studies, seven studies assessed the ORR of ICls combined with radiotherapy(a). The pooled DCR for the six studies is shown in (b). Six studies provided the PFSR6m and seven provided the OSR1y, which are shown in (c) and (d).

a

study

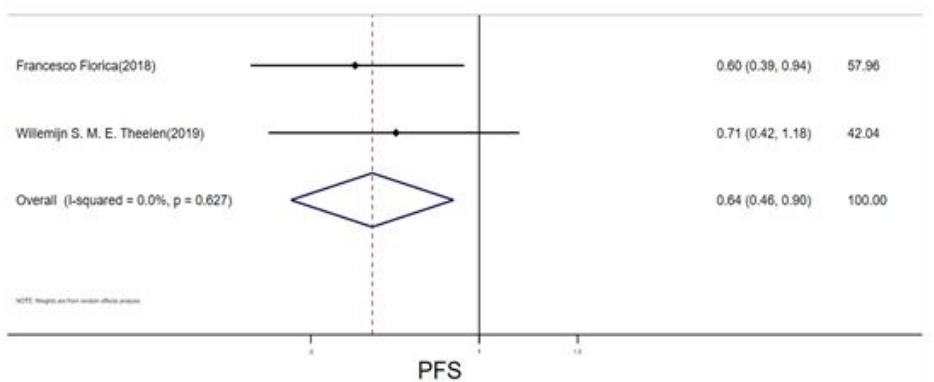

Study

'HR' (95\% Cl) Weighte

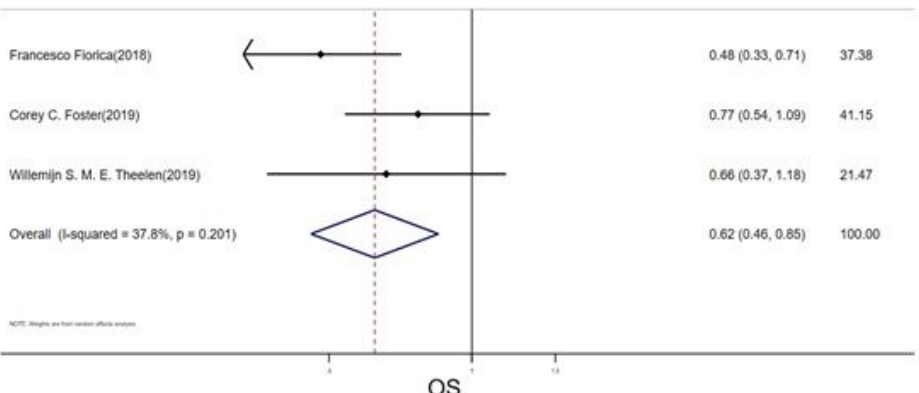

OS

Figure 3

Forest plot of the combined HR values of PFS and OS of two-armed studies. Among the ten studies, two studies assessed the HR values of PFS(a). The combined HR values of OS for the three studies is shown in (b). 